\title{
A positron-emission tomography (PET)/magnetic resonance imaging (MRI) platform to track in vivo small extracellular vesicles
}

striReceived 00th January 20xx, Accepted 00th January 20xx

DOI: $10.1039 / \times 0 \times x 00000 x$

\author{
Arnab Banerjee ${ }^{a}$, Vitor Alves, Tiago Rondão ${ }^{b}, J^{\prime}$ sé Sereno $^{c}$, Ângela Neves ${ }^{c}$, Miguel Lino $^{a}$, Andreia
} Ribeiro ${ }^{a}$, Antero J. Abrunhosa ${ }^{c}$ and Lino S. Ferreira ${ }^{a, b^{*}}$
Here we report a two-step surface modification methodology to radiolabel small extracellular vesicles (SEVs) with ${ }^{64} \mathrm{CuCl}_{2}$ for PET/MRI imaging. The modification did not change or damage the morphology, surface receptor proteins and internal RNA content. Radiolabeled SEVs could be detected in organs with low accumulation such as the brain (0.4-0.5\% ID/g) and their brain location determined by MRI.

SEVs are nanovesicles, with sizes ranging between 30 and 200 $\mathrm{nm}$, secreted by cells. ${ }^{1}$ They contain biomolecules (mRNA, miRNA, proteins and lipids) important for cell-cell communication. There is an increasing interest in using these SEVs for diagnostic (e.g. early detection of cancer, myocardial infarction, stroke) $)^{2-4}$ but also for regenerative medicine applications. ${ }^{5,6}$ In the latter case, SEVs isolated from mesenchymal stromal cells (MSCs), ${ }^{7,8}$ umbilical cord blood $^{6,9}$ as well as other cell/tissues have been tested for the treatment of ischemic diseases including stroke, ${ }^{7,8}$ myocardial infarction ${ }^{5,6}$ and chronic wounds. ${ }^{9}$ In addition, SEVs have been engineered to act as delivery systems of siRNA and miRNAs to interfere with tissue biology. ${ }^{8}$ Therefore, methods to track SEVs in vivo and follow their biodistribution are very important to fully evaluate their regenerative potential.

Fluorescence, ${ }^{10,11}$ luminescence, ${ }^{10,11}$ magnetic resonance imaging (MRI), ${ }^{12,}{ }^{13}$ computed tomography ${ }^{14}$ and single-photon emission computed tomography (SPECT) ${ }^{15,16}$ imaging techniques have been used to monitor in vivo SEVs. Fluorescence and luminescence techniques are relatively easy to operate, readily accessible in research labs and can provide spatiotemporal distribution of SEVs, however, they do not offer high sensitivity and absolute quantification. An alternative to fluorescence/luminescence techniques is MRI which offers anatomic information. In this case, SEVs are typically labelled with superparamagnetic iron oxide nanoparticles with sizes between 5 and $150 \mathrm{~nm} .{ }^{12,13,17}$ Unfortunately, MRI does not have enough sensitivity for the low concentration (normally $10 \mu \mathrm{M}$ to $10 \mathrm{mM}$

\footnotetext{
a. CNC-Center for Neurosciences and Cell Biology, University of Coimbra, 3004-517 Coimbra, Portugal.

${ }^{b .}$ Faculty of Medicine, University of Coimbra, 3004-504 Coimbra, Portugal.

c. ICNAS/CIBIT - Institute for Nuclear Sciences Applied to Health; University of Coimbra; 3000-548 Coimbra, Portugal

+ Footnotes relating to the title and/or authors should appear here

Electronic Supplementary Information (ESI) available: [details of any supplementary information available should be included here]. See
} DOI: $10.1039 / x 0 x x 00000 x$ contrast agent was needed) ${ }^{18}$ of SEVs typically accumulated in tissue. Computed tomography requires the labelling of SEVs with inorganic nanoparticles which may accumulate in tissues. ${ }^{14}$ Alternatively, SPECT/CT offers sensitivity; however, the acquisition and quantification of the images raises some technical issues and the stability and bioactivity of the labelled SEVs have not been demonstrated. ${ }^{15,16}$ PET imaging is a very interesting alternative to SPECT for SEV tracking as PET is superior to SPECT regarding sensitivity (approximately 2 to 3 orders of magnitude) ${ }^{19}$ and provides quantitative images. During the submission of this study, a PET imaging technique for SEV tracking has been reported. ${ }^{20}$ The study used ${ }^{124}$ I, with a half-life over 4 days, which was reacted covalently with the tyrosine aminoacids of proteins in the SEV membrane. PET imaging was combined with computed tomography (CT) to include anatomical details. Despite the significant progresses, the effect of iodine in the properties of EVs (e.g. intravesicle content, cell internalization, SEV bioactivity), the control of radiolabelling (EVs from different cell sources may have significant differences in protein content at the membrane) as well as anatomical information about the accumulation of EVs in organs with low accumulation (e.g. brain) was not investigated.

Here, we describe a PET/MRI imaging platform to track in vivo SEVs and we demonstrate the effect of the radiotracer in the properties of SEVs. We have selected ${ }^{64} \mathrm{Cu}^{2+}\left(\mathrm{t}_{1 / 2}=12.7 \mathrm{~h}, \mathrm{I}_{\beta+}=17 \%, \mathrm{I}_{\beta-}\right.$ $=39 \%, \mathrm{IEC}=43 \%, \mathrm{E}_{\max }=0.656 \mathrm{MeV}$ ) as radiotracer because it is a widely used radionuclide in diagnostic as well as radiotherapy applications, and can be produced in large scale with high specific activity. ${ }^{21,22}{ }^{64} \mathrm{Cu}^{2+}$ was conjugated to $\mathrm{SEV}$ s in two steps. The surface of SEVs was initially conjugated with the metal chelator $1,4,7,10$ tetraazacyclododecane-1,4,7,10-tetraacetic acid (DOTA) followed by the complexation of the DOTA with ${ }^{64} \mathrm{CuCl}_{2}$ (Fig. 1). DOTA was selected to complex the ${ }^{64} \mathrm{Cu}^{2+}$ because it forms kinetically and thermodynamically stable copper complex which is stable enough for in vivo conditions. ${ }^{23,24}$ The conjugated SEVs (SEV-DOTA-Cu) were then evaluated for morphology (size and charge), stability in human serum and bioactivity against human cells. Finally, we demonstrate in vivo the utility of SEV-DOTA-Cu by monitoring its biodistribution in a mouse animal model. 


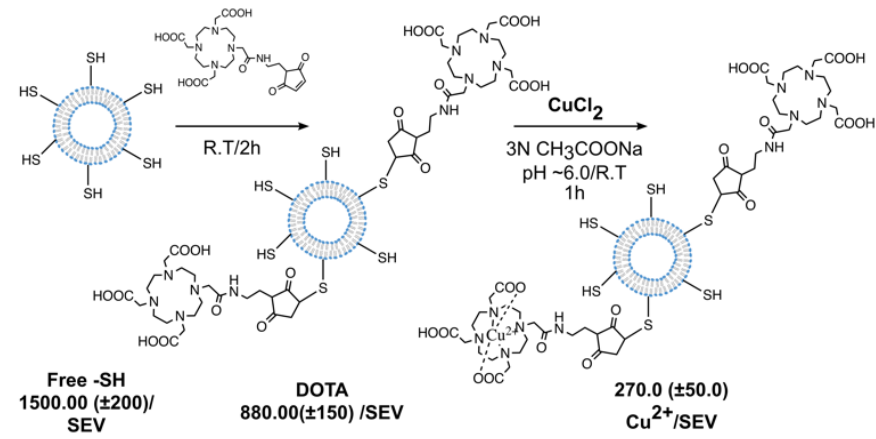

Figure 1. Schematic representation of the SEV radiolabelling process. DOTA derivative with a maleimide group in one arm was used as a metal chelator. Maleimide was reacted with the free surface thiol group of SEV. ${ }^{64} \mathrm{CuCl}_{2}$ was used as radiolabelling agent.

In this study, we have isolated SEVs from human umbilical cord blood mononuclear cells (hUCB-MNCs) cultured under hypoxic conditions. We selected hUCB-MNCs because these cells were relatively easy to obtain from banks and the enhanced bioactivity of SEVs collected from neonatal cells has been demonstrated relatively to adult cells. ${ }^{25}$ In addition, we cultured hUCB-MNCs in hypoxic conditions because recent studies have shown that SEVs were more bioactive than SEVs isolated from cells cultured under normoxic conditions. ${ }^{26}$ SEVs were purified using a sequential

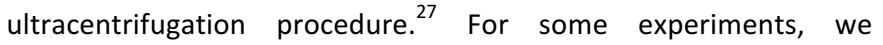
performed a size exclusion chromatography to remove protein aggregates. ${ }^{28,}{ }^{29}$ SEVs were then characterized by dynamic light scattering (DLS; Figs. $2 \mathrm{~B}$ and $2 \mathrm{C}$ ), nanoparticle tracking analyses (NTA; Figs. S1A, S1B and S1C) protein quantification, flow cytometry analyses (Fig. 2D) and instant thin layer chromatography (ITLC) (Fig. $2 E)$. In this last case, SEVs were characterized for the expression of the EV markers $\mathrm{CD} 9$ and $\mathrm{CD} 63^{1,30}$ as well as the hematopoietic marker CD45 (Fig. 2D). Overall, our results showed that SEVs had an average diameter of $100 \mathrm{~nm}$, a zeta potential of $-34 \mathrm{mV}$, and expressed high levels of EV markers including CD9 and CD63 as well as the hematopoietic marker CD45.
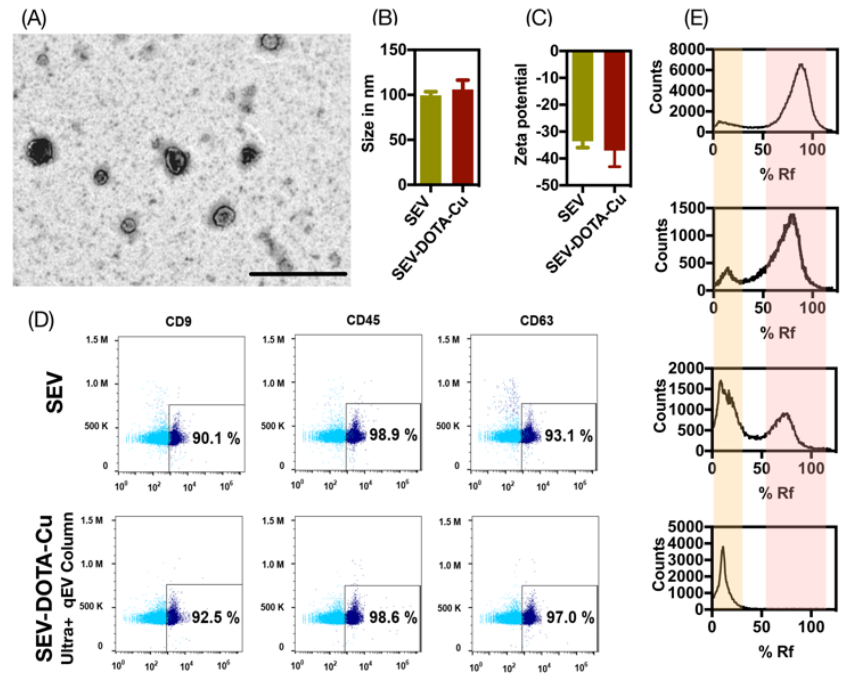

Figure 2. Characterization of SEV-DOTA-Cu. (A) Representative TEM picture of SEV-DOTA-Cu. Bar corresponds to $500 \mathrm{~nm}$. (B) Size and (C) zeta potential analyses of SEV-DOTA-Cu by DLS. Results are average $\pm S E M, n=3$. (D) Expression of surface markers in SEVs and SEV-DOTA-Cu by flow cytometry. Light blue represents SEVs stained with IgG control while dark blue show SEVs stained with CD9, CD45 or CD63. (E) ITLC measurement to monitor the purity of SEV-DOTA-Cu. (i) Free $\mathrm{CuCl}_{2}$, (ii) SEV-DOTA mixed with $\mathrm{CuCl}_{2}$ at $\mathrm{O}$ h, (iii) SEV-DOTA mixed with $\mathrm{CuCl}_{2}$ at $1 \mathrm{~h}$ before purification, (iv) SEV-DOTA-Cu after purification.

To conjugate the metal chelator DOTA at the surface of SEVs, we have used SEV free thiol groups. The concentration of free thiol groups per SEV was $1,500( \pm 200)$, as quantified by a thiol fluorescent probe (see Materials and Methods). Next, the free thiol groups in the surface of SEVs were reacted with the maleimide group present in the DOTA molecule to form a stable covalent bond (Fig. 1) with a yield of approx. 50-60\%. At the end of reaction, 880 ( \pm 150$)$ DOTA were conjugated per SEV. Finally, SEV-DOTA were complexed with ${ }^{64} \mathrm{CuCl}_{2}$ and purified by spin columns (see Materials and Methods) to eliminate the non-complexed ligand. SEV-DOTA complexed $270( \pm 50.0) \mathrm{Cu}^{2+}$ per SEV, and thus presented a complexation efficiency of approximately $30 \%$. For our studies, we have used 6.2 to $40.0 \mu \mathrm{Ci}$ activity per $1 \times 10^{9}$ particles of SEVs with radiochemical yield $(\mathrm{RCY}) \sim 15-33 \%$. Yet, this activity can be increased to $\mathrm{mCi}$ (corresponding to 9 nanomolar of $\mathrm{Cu}^{2+}$ ) per $1 \times 10^{9}$ particles of SEVs, in case a high concentration of ${ }^{64} \mathrm{CuCl}_{2}$ was used. This level of radioactivity was not possible using a previous methodology to radiolabel SEVs with ${ }^{99 \mathrm{~m}} \mathrm{Tc}$-HMPAO for SPECT imaging. ${ }^{15}$

The radiochemical purity of SEV-DOTA-Cu before and after purification by spin column was characterized by ITLC. The free ${ }^{64} \mathrm{CuCl}_{2}$ moved along the TLC strip whereas SEVs stayed in the bottom of the strip (Fig. 2E). SEV-DOTA-Cu, after purification by spin column, showed only one band (and thus a purity of $\approx 100 \%$ ) corresponding to the presence of modified SEVs. This level of purity compares favourably with a previous strategy of radiolabelling SEVs by the entrapment of the radioligand in the interior of the vesicle ${ }^{15}$ (purity of $93.7 \%$ ). In our case, the high level of purity was due to the purification methodology adopted as well as the stability of the bond between the DOTA and ${ }^{64} \mathrm{Cu}^{2+}$.

The morphology, size and charge of purified SEVs-DOTA-Cu were characterized by transmission electron microscopy (TEM) (Fig. 2A), DLS (Fig. 2B), zeta potential (Fig. 2C) and NTA (Fig. S1A and $\mathrm{S} 1 \mathrm{~B})$. TEM results showed a typical cup shaped morphology as well as the presence of a bilayer membrane indicating that the morphology of SEVs was not significantly altered after conjugation (Fig. 2A). No statistical changes in size and zeta potential were observed between original SEVs and SEV-DOTA-Cu, as evaluated by DLS (Figs. 2B and 2C) and NTA analyses (Fig. S1A, S1B). In addition, no signs of aggregation of SEVs-DOTA-Cu were observed at room temperature even after $24 \mathrm{~h}$ post-reaction (Fig. S1B and S1C). This contrasts with previous attempts of SEV surface radiolabelling with SCN-Bn-NOTA and ${ }^{68} \mathrm{Ga}$, in which aggregation of SEVs was described. ${ }^{15}$ Moreover, the content of surface proteins in SEVs such as CD63 and CD9 (Fig. 2D) was not affected by the labelling procedure as well as the microRNA content (Fig. S2A, S2B). Therefore, our results demonstrate the feasibility to label SEVs in 
the surface. This surface labelling strategy prevents also potential interactions of metal ions with proteins and non-coding RNAs entrapped in the SEVs.

Next, we evaluated whether the bioactivity of SEVs was affected by the $\mathrm{Cu}$ labelling procedure. Previous studies have shown that SEVs isolated from different type of cells might have a prosurvival effect in cells cultured under ischemic conditions. ${ }^{31,32}$ Therefore, we evaluated the pro-survival activity of both SEVs and SEV-DOTA-Cu against ischemic endothelial cells. Human umbilical vein endothelial cells (HUVECs) were cultured for $2 \mathrm{~h}$ in ischemic conditions (culture media without glucose, in the absence of oxygen) and then treated with SEVs $\left(4.5 \times 10^{9}\right.$ particles $/ \mathrm{mL}$, without $\mathrm{Cu}$ labelling) for $48 \mathrm{~h}$ in normoxic conditions and full cell culture media. Cells treated with SEV showed a higher viability (as evaluated by cell counting) than non-treated cells (Fig. 3B). Interestingly, HUVECs treated with SEV-DOTA-Cu, in the same set up described before, showed a higher viability than cells treated with SEVs without copper. We further checked the internalisation of SEVs with and without modification (Fig. 3C, 3D). Confocal Zstacks reveal that SEVs are internalised by HUVEC in both cases. Analysis of cell fluorescence showed no significant difference after the modification with DOTA-Cu. These results suggest that the higher bioactivity of SEV-DOTA-Cu is not caused by higher internalization. A possible explanation for this is related to the proangiogenic effect of copper, ${ }^{33},{ }^{34}$ whose role in the survival of endothelial cells has been previously demonstrated, particularly in reduced nutrient conditions. ${ }^{35}$ Therefore, our labelling strategy may contribute for the bioactivity of SEVs.
(A)

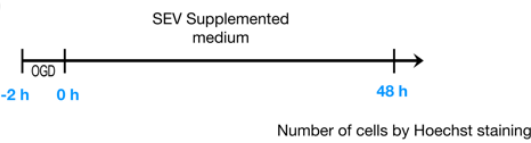

Number of cells by Hoechst staining

(C)

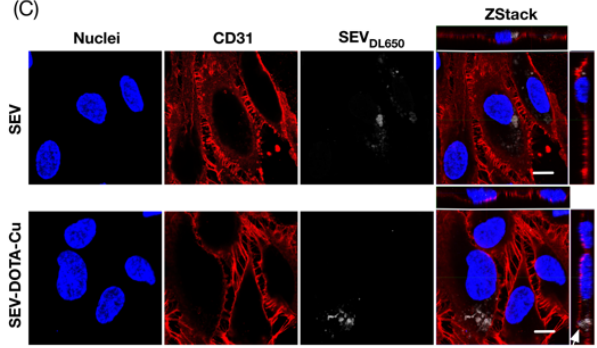

(B)

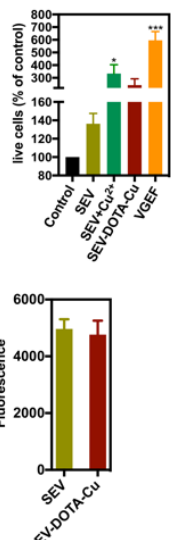

Figure 3. Internalization and bioactivity of SEVs and SEV-DOTA-Cu against human endothelial cells. (A) Bioactivity experiment protocol. OGD stands for oxygen-glucose deprivation. (B) Bioactivity of SEV and SEV-DOTA-Cu against human endothelial cells. Endothelial cells were cultured for $2 \mathrm{~h}$ in ischemic conditions for $2 \mathrm{~h}$ and then treated with SEVs or SEV-DOTA-Cu for $48 \mathrm{~h}$ in normoxic conditions and glucose supplemented media. Live cells were counted after staining cell nuclei with Hoechst dye. SEV or SEV-DOTACu concentration was $4.5 \times 10^{9}$ particles per $\mathrm{mL}$ of cell culture media. $4.5 \times 10^{9}$ particles of SEV-DOTA-Cu had $40 \mathrm{nM} \mathrm{Cu}^{2+}$. $\mathrm{SEV}+\mathrm{Cu}^{+2}$ had also $4.5 \times 10^{9}$ particles $+40 \mathrm{nM} \mathrm{Cu}^{+2}$ in $1 \mathrm{~mL}$. Results are average $\pm S E M, n=3$. Statistical analysis was performed by One-way ANOVA followed by a Tukey's multiple comparisons test. (C) Internalization of the SEV and SEV-DOTA-Cu in human endothelial cells. The white arrow indicate the internalization of the SEVs. Bar corresponds to $10 \mu \mathrm{m}$. (D) Fluorescence signal after SEV internalization by the endothelial cells (please see supplementary information for the quantifications).
For in vivo applications, it is very important that the copper labelling is stable and the ligand does not leach overtime from the chelator. Therefore, we evaluated the stability of the copper labelling after suspending SEVs-DOTA-Cu in human serum for $24 \mathrm{~h}$. As control, we suspended SEVs-DOTA-Cu in PBS. At different times, a fraction of the suspension was characterized by ITLC (Fig. S3A). The results showed that SEVs-DOTA-Cu were stable in serum and PBS (94\% and 97\%, respectively), at least for $24 \mathrm{~h}$. The stability of SEV-DOTA-Cu was also evaluated in the animal blood. For this purpose, SEVs-DOTA-Cu were administered intravenously in mice and after 1 or $4 \mathrm{~h}$ blood samples collected and characterized by ITLC (Fig. S3B). Our results indicated that more than $95 \%$ of SEV-DOTA$\mathrm{Cu}$ was observed as a complex, without the leaching of ${ }^{64} \mathrm{Cu}^{2+}$ from the chelator.

The biodistribution of SEV-DOTA-Cu (100-150 $\mu \mathrm{Ci} ; 2.5 \times 10^{10}$ $3.5 \times 10^{10}$ ) and DOTA-Cu was evaluated after intravenous administration in the tail vein of $\mathrm{C} 57 \mathrm{BL} / 6 \mathrm{~J}$ mice. At different time points, animals were sacrificed and the organs collected for radioactivity quantification. Animals administered with DOTA-Cu showed almost no radioactivity after $1 \mathrm{~h}$, indicating a rapid elimination from the animal body (Fig. S4A), whereas SEV-DOTA-Cu showed still a significant radioactivity after $3 \mathrm{~h}$. In this last case, the highest level of radioactivity in all the organs was found at $2 \mathrm{~h}$. At this time point, animals presented the highest radioactivity in liver ( 30\%; Fig. 4E) followed by lung, kidney, bowel and stomach. The accumulation in the brain was the lowest ( 0.4-0.5 \%) likely due to the selective permeability of the blood brain barrier.

(A)

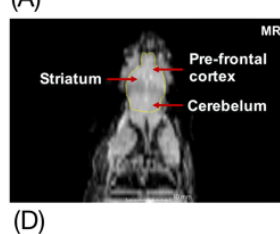

(B)

(C)

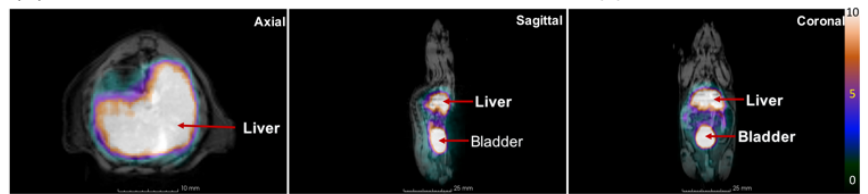

(G)

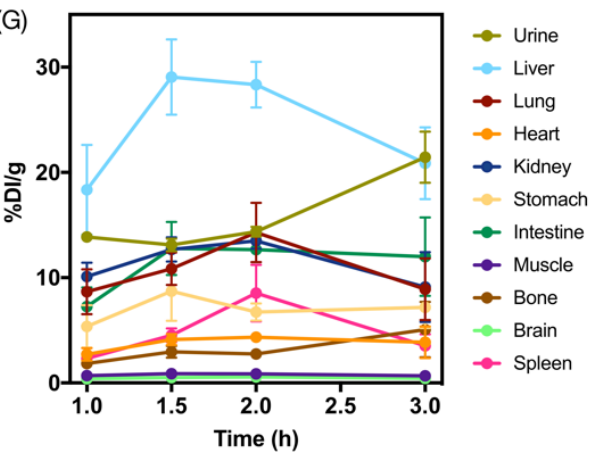

Figure 4. Biodistribution of SEV-DOTA-Cu in mice. (A-C) Representative MRI, PET and MRI/PET images showing the accumulation of SEVs in the brain. Brain was scanned during 20-60 min after EV injection. (D-F) Representative mouse PET/MRI images taken $\sim 3 \mathrm{~h}$ post-administration of EVs. (F) Biodistribution of SEV-DOTA-Cu in mice. Mice were sacrificed at $1 \mathrm{~h}, 1.5 \mathrm{~h}, 2$ $\mathrm{h}$ and $3 \mathrm{~h}$ after intravenous injection of SEV-DOTA-Cu. Radioactivity was 
measured for quantitative analysis of biodistribution. Results are average \pm SEM, $n=3-4$.

Next, mice administered intravenously with SEV-DOTA-Cu $(n=2$ mice) and DOTA-Cu (control; $n=1$ mice) and then imaged by PETMRI. All PET scans were performed using a prototype of a highacceptance small-animal PET based on resistive plate chambers (RPC-PET). ${ }^{36}$ The whole body of a mice was scanned in three sections (liver-heart, brain, kidney- bladder) for $3 \mathrm{~h}$ and $40 \mathrm{~min}$. Images were reconstructed using OSEM algorithm and cubic voxel of $0.5 \mathrm{~mm}$ width. Height fiducial markers that can be viewed both in the PET and MRI imaging were placed in the mice bed and MRI imaging was done after PET without moving the mice from the bed, under anesthesia. Our results clear indicate the accumulation of SEVs in the liver. The brain (the organ with the lowest accumulation) showed homogeneous accumulation of SEVs. In detail, high resolution RPC-PET images analysis revealed similar uptake of the radiolabel-SEVs in the striatum, pre-frontal cortex and cerebellum (period 20 to $60 \mathrm{~min}$ : $0.96,0.91$ and $1.00 \% \mathrm{ID} / \mathrm{g}$, respectively), compared to the late acquisition (period $3 \mathrm{~h}$ to $3 \mathrm{~h} 40$ : $0,86,0.81$ and $0.78 \% \mathrm{ID} / \mathrm{g}$, respectively). Interestingly no activity was observed in the salivary gland after $3 \mathrm{~h}$ injection of SEV-DOTA$\mathrm{Cu}$ further supporting the stability of our surface modification strategy over the encapsulation strategy where significant amount of activity in the salivary gland was observed after $3 \mathrm{~h}$ of injection due to the leaching of ${ }^{99 \mathrm{~m}} \mathrm{Tc}^{15}$

Overall, we have developed a SEV radiolabeling strategy useful for PET/MRI imaging. The PET/MRI imaging platform offers several advantages relatively to SPECT/CT or PET/CT imaging platforms. MRI offers better anatomical details than CT images. This is of utmost importance to investigate the biological effect of SEVs in complex organisms. In addition, PET imaging offers better resolution than SPECT imaging. The sensitivity of PET scanners is higher because of the geometry of the scanners, which surround the body with a ring of detectors, whereas the SPECT system is limited by a physical collimator and requires planar detectors to rotate around the body to acquire a tomographic image. ${ }^{37}$ PET scanners detect 100 time more emitted photons than SPECT scanners. ${ }^{19}$ The higher sensitivity of the PET scanner allows the administration of a lower activity of radiotracers. In the current study, we have successfully performed the PET imaging after injecting $180 \mu \mathrm{Ci}$, with similar sensitivity to the one obtained with higher copper activity $(665 \mu \mathrm{Ci})$. Using the current radiolabelling strategy and equipment set up, one can detect as low as $1 \times 10^{5}$ $1 \times 10^{6}$ particles per tissue. Most importantly our surface modification strategy didn't compromise either the surface receptor proteins or internal miR content of SEVs which was not tested in previous studies. SEV-DOTA-Cu were $94 \%$ stable in serum and didn't form aggregates.

PET imaging and copper activity measurements by well counting showed that hUCB-MNC SEVs have a preferential accumulation in the liver that peaked at $2 \mathrm{~h}$ post-intravenous administration. Previous studies, showed that SEVs isolated from human embryonic kidney cell lines, ${ }^{11}$ mouse liver cell lines, ${ }^{20}$ primary human mesenchymal stem cells ${ }^{38}$ and mouse macrophages ${ }^{15}$ accumulated preferentially in liver, ${ }^{15,16}$ spleen $^{11}$ or bladder, ${ }^{20}$ in general $3 \mathrm{~h}$ postintravenous administration. ${ }^{11,15}$ The differences between our and previous studies might be due to differences in the nature of SEVs. Liposomes larger than $160 \mathrm{~nm}$ accumulate preferentially in the spleen than in the liver. ${ }^{11,} 39$ The decrease in liposome size decreases the accumulation in spleen and increases in the liver. Because our SEVs have an average diameter of $114 \mathrm{~nm}$ it is likely that they are more prone to accumulate in the liver. The presence of SEV-DOTA-Cu in the urine was less than $10 \%$ after $1 \mathrm{~h}$ postadministration which gradually increased along time and after $3 \mathrm{~h}$ we had $20-25 \%$ in urine which indicates the elimination of the SEVDOTA-Cu from the body.

The use of copper as a radiotracer opens new opportunities to couple the bioactivity of copper with the bioactivity of proteins and non-coding RNAs within SEVs. In the context of Regenerative Medicine, it has been demonstrated that copper has an antioxidant activity, is able to stimulate the proliferation of human endothelial cells ${ }^{40}$ and is able to regulate the expression of claudins and thus control blood brain barrier permeability. ${ }^{41}$ In addition, copper is very important for brain activity and recent studies indicate that the disturbance on brain copper homeostasis may lead to Alzheimer's disease. ${ }^{42}$ Therefore, the use of copper as a radiotracer opens new opportunities in terms of regenerative and therapeutic medicine.

\section{Conclusions}

We report a methodology to radiolabel SEVs with ${ }^{64} \mathrm{CuCl}_{2}$ for $\mathrm{PET} / \mathrm{MRI}$ imaging. The methodology includes the initial conjugation of a metal chelator on the surface of SEVs followed by the complexation of the modified SEVs with ${ }^{64} \mathrm{CuCl}_{2}$. SEV-DOTA-Cu had similar diameter (114 $\mathrm{nm}$ ) and zeta potential (-33.62 versus -37.06) as the non-conjugated ones, showed stable radiolabeling (ca. 94\% of the signal was maintained) for $24 \mathrm{~h}$ when vesicles were suspended in 1:1 serum:buffer, and demonstrated a higher bioactivity against human endothelial cells as non-conjugated vesicles due to the angiogenic properties of $\mathrm{Cu}$. It was also confirmed that the cell-internalization and internal miR content of the SEVs was not affected by their surface modification. The biodistribution of SEV-DOTA-Cu could be monitored by PET following intravenous administration in mice. Two hours' postadministration, SEV-DOTA-Cu showed the highest accumulation in the liver $(25-30 \%)$ and the lowest in the brain (0.4-0.5\%). The labelling strategy presented here may be useful for diagnostics and therapies based in SEVs since it is relatively simple and very sensitive.

\section{Acknowledgements}

The authors would like to thank the financial support of Project TRoMBONE grant number 748583 under the Horizon 2020 program of European commission, "StrokeTherapy" co-promoted by Stemlab, Rovisco Pais and Universidade de Coimbra, POCI-01 -0247FEDER-003386), EC project ERAatUC (Ref:669088) and FCT project (Ref no. POCI-01-0145-FEDER-029919). 


\section{Conflicts of interest}

No conflict of interest to declare.

\section{Notes and references}

1. C. Théry, L. Zitvogel and S. Amigorena, Nature Reviews Immunology, 2002, 2, 569.

2. J. Wang, Y. Zheng and M. Zhao, Frontiers in Pharmacology, 2017, 7.

3. L. Barile, V. Lionetti, E. Cervio, M. Matteucci, M. Gherghiceanu, L. M. Popescu, T. Torre, F. Siclari, T. Moccetti and G. Vassalli, Cardiovascular Research, 2014, 103, 530-541.

4. H. Xin, Y. Li, Z. Liu, X. Wang, X. Shang, Y. Cui, Z. G. Zhang and M. Chopp, Stem cells, 2013, 31, 2737-2746.

5. S. Sahoo, E. Klychko, T. Thorne, S. Misener, K. M. Schultz, M. Millay, A. Ito, T. Liu, C. Kamide, H. Agrawal, H. Perlman, G. Qin, R. Kishore and D. W. Losordo, Circ Res, 2011, 109, 724-728.

6. P. Mathiyalagan, Y. Liang, D. Kim, S. Misener, T. Thorne, C. E. Kamide, E. Klyachko, D. W. Losordo, R. J. Hajjar and S. Sahoo, Circ Res, 2017, 120, 1466-1476

7. H. Xin, Y. Li, Y. Cui, J. J. Yang, Z. G. Zhang and M. Chopp, J Cereb Blood Flow Metab, 2013, 33, 1711-1715.

8. H. Xin, M. Katakowski, F. Wang, J. Y. Qian, X. S. Liu, M. M. Ali, B. Buller, Z. G. Zhang and M. Chopp, Stroke, 2017, 48, 747-753.

9. Y. Hu, S. S. Rao, Z. X. Wang, J. Cao, Y. J. Tan, J. Luo, H. M. Li, W. S. Zhang, C. Y. Chen and H. Xie, Theranostics, 2018, 8, 169-184.

10. T. Tian, H.-X. Zhang, C.-P. He, S. Fan, Y.-L. Zhu, C. Qi, N.-P. Huang, Z.-D. Xiao, Z.-H. Lu and B. A. Tannous, Biomaterials, 2018, 150, 137-149.

11. C. P. Lai, O. Mardini, M. Ericsson, S. Prabhakar, C. A Maguire, J. W. Chen, B. A. Tannous and X. O. Breakefield, ACS nano, 2014, 8, 483-494.

12. A. Busato, R. Bonafede, P. Bontempi, I. Scambi, L. Schiaffino, D. Benati, M. Malatesta, A. Sbarbati, P. Marzola and R. Mariotti, International journal of nanomedicine, 2016, 11, 2481.

13. L. Hu, S. A. Wickline and J. L. Hood, Magnetic resonance in medicine, 2015, 74, 266-271.

14. O. Betzer, N. Perets, A. Angel, M. Motiei, T. Sadan, G. Yadid, D. Offen and R. Popovtzer, ACS nano, 2017, 11, 10883-10893.

15. D. W. Hwang, H. Choi, S. C. Jang, M. Y. Yoo, J. Y. Park, N. E. Choi, H. J. Oh, S. Ha, Y. S. Lee, J. M. Jeong, Y. S. Gho and D. S. Lee, Sci Rep, 2015, 5, 15636.

16. Z. Varga, I. Gyurko, K. Paloczi, E. I. Buzas, I. Horvath, N. Hegedus, D. Mathe and K. Szigeti, Cancer Biother Radio, 2016, 31, 168-173.

17. J. L. Hood, M. J. Scott and S. A. Wickline, Analytical biochemistry, 2014, 448, 41-49.

18. S. Sinharay and M. D. Pagel, Annual Review of Analytical Chemistry, 2016, 9, 95-115.

19. A. Rahmim and H. Zaidi, Nuclear medicine communications, 2008, 29, 193-207.

20. F. Royo, U. Cossio, A. Ruiz de Angulo, J. Llop and J. M. Falcon-Perez, Nanoscale, 2019, 11, 1531-1537.

21.
22.

D. W. McCarthy, R. E. Shefer, R. E. Klinkowstein, L. A. Bass, W. H. Margeneau, C. S. Cutler, C. J. Anderson and M. J. Welch, Nuclear medicine and biology, 1997, 24, 35-43.

23. M. K. Moi, C. F. Meares, M. J. McCall, W. C. Cole and S. J. DeNardo, Analytical biochemistry, 1985, 148, 249-253.

24. W. C. Cole, S. J. DeNardo, C. F. Meares, M. J. McCall, G. L. DeNardo, A. L. Epstein, H. A. O'Brien and M. K. Moi, International Journal of Radiation Applications and Instrumentation. Part B. Nuclear Medicine and Biology, 1986, 13, 363-368.

25

U. Agarwal, A. George, S. Bhutani, S. Ghosh-Choudhary, J. T. Maxwell, M. E. Brown, Y. Mehta, M. O. Platt, Y. Liang, S. Sahoo and M. E. Davis, Circ Res, 2017, 120, 701-712.

26. W. D. Gray, K. M. French, S. Ghosh-Choudhary, J. T. Maxwell, M. E. Brown, M. O. Platt, C. D. Searles and M. E. Davis, Circ Res, 2015, 116, 255-263.

C. Thery, S. Amigorena, G. Raposo and A. Clayton, Curr Protoc Cell Biol, 2006, Chapter 3, Unit 322.

28. R. J. Lobb, M. Becker, S. Wen Wen, C. S. Wong, A. P. Wiegmans, A. Leimgruber and A. Möller, Journal of extracellular vesicles, 2015, 4, 27031.

29. A. N. Böing, E. Van Der Pol, A. E. Grootemaat, F. A. Coumans, A. Sturk and R. Nieuwland, Journal of extracellular vesicles, 2014, 3, 23430.

30. J. C. Akers, D. Gonda, R. Kim, B. S. Carter and C. C. Chen, Journal of Neuro-Oncology, 2013, 113, 1-11.

31. Y. Feng, P. G. Rhodes and A. J. Bhatt, Pediatric research, 2008, 64, 370.

32

D. C. Pedroso, A. Tellechea, L. Moura, I. Fidalgo-Carvalho, J. Duarte, E. Carvalho and L. Ferreira, PloS one, 2011, 6, e16114.

B. McAuslan and W. Reilly, Experimental cell research, 1980, 130, 147-157.

34. H. Xie and Y. J. Kang, Current medicinal chemistry, 2009, 16, 1304-1314.

35. C. Stähli, N. Muja and S. N. Nazhat, Tissue Engineering Part A, 2012, 19, 548-557.

36

P. Martins, A. Blanco, P. Crespo, M. F. F. Marques, R. F. Marques, P. M. Gordo, M. Kajetanowicz, G. Korcyl, L. Lopes and J. Michel, Journal of Instrumentation, 2014, 9, C10012.

R. J. Hicks and M. S. Hofman, Nature reviews Clinical oncology, 2012, 9, 712.

O. P. Wiklander, J. Z. Nordin, A. O'Loughlin, Y. Gustafsson, G. Corso, I. Mäger, P. Vader, Y. Lee, H. Sork and Y. Seow, Journal of extracellular vesicles, 2015, 4, 26316.

39. R. Abra and C. A. Hunt, Biochimica et Biophysica Acta (BBA)-Lipids and Lipid Metabolism, 1981, 666, 493-503.

40. G. F. Hu, J Cell Biochem, 1998, 69, 326-335.

41. J. Wang, J. Chen, Z. Tang, Y. Li, L. Hu and J. Pan, Biol Trace Elem Res, 2016, 174, 132-141.

42. I. F. Scheiber, J. F. Mercer and R. Dringen, Prog Neurobiol, 2014, 116, 33-57. 